\title{
Article
}

\section{Peripheral and Integral Binding of Cytochrome $c$ to Phospholipids Vesicles}

Silke Oellerich, Sophie Lecomte, Maite Paternostre, Thomas Heimburg, and Peter Hildebrandt

J. Phys. Chem. B, 2004, 108 (12), 3871-3878• DOI: 10.1021/jp036799t • Publication Date (Web): 02 March 2004

\section{Downloaded from http://pubs.acs.org on March 24, 2009}

\section{More About This Article}

Additional resources and features associated with this article are available within the HTML version:

- Supporting Information

- $\quad$ Links to the 2 articles that cite this article, as of the time of this article download

- $\quad$ Access to high resolution figures

- $\quad$ Links to articles and content related to this article

- $\quad$ Copyright permission to reproduce figures and/or text from this article

\section{View the Full Text HTML}

\section{ACS Publications}




\title{
Peripheral and Integral Binding of Cytochrome $c$ to Phospholipids Vesicles
}

\author{
Silke Oellerich, ${ }^{\dagger, \#}$ Sophie Lecomte, ${ }^{\ddagger}$ Maite Paternostre, ${ }^{\S}$ Thomas Heimburg," and \\ Peter Hildebrandt*, $\perp$
}

Max-Planck-Institut für Bioanorganische Chemie, Stiftstr. 34-36, 45470 Mülheim, Germany,Laboratoire de Dynamique, Interactions et Réactivité, UMR-7075, CNRS-Université Paris VI, 2 Rue Henri Dunant,

F-94320 Thiais, France,Laboratoire des proteins transtuctrices d'energie, URA 2096, CEA-CNRS, CEA-Saclay, F-91191 Gif/Yvette, France,Max-Planck-Institut für biophysikalische Chemie, Am Fassberg 11, D-37077 Göttingen, Germany, andTechnische Universität Berlin, Institut für Chemie, Max-Volmer-Laboratorium, Strasse des 17. Juni 135, D-10623 Berlin, Germany

Received: September 18, 2003; In Final Form: January 23, 2004

\begin{abstract}
The interactions of ferric cytochrome $c(\mathrm{Cyt}-c)$ with dioleoyl-phosphatidylglycerol (DOPG) at low ionic strength have been studied by viscosity and turbidity measurements as well as by resonance Raman, circular dichroism, and UV-vis-absorption spectroscopy to monitor the structural changes of the liposomes and the protein upon complex formation. The observed mutual structural changes in the liposomes and the protein are associated with three different modes of protein binding. At high lipid/protein (L/P) ratios, Cyt-c binds electrostatically to the anionic headgroups of the phospholipids which induces structural changes of the protein. Decreasing the L/P-ratio weakens the electrostatic interactions such that membrane anchoring of Cyt- $c$ can effectively compete with peripheral binding. This mode of binding is accompanied by an increase of long-range liposomeliposome interactions. Upon lowering the L/P-ratio below the ratio for full protein coverage of the vesicles, further Cyt-c binding is achieved via interactions with the protein monolayer. This mode of binding initiates phase separation of the liposome aggregates from the aqueous buffer. Our results indicate that the crucial parameter controlling the interplay between the binding modes appears to be the membrane surface potential which in turn sensitively depends on the protein coverage.
\end{abstract}

\section{Introduction}

Interactions of soluble proteins with membranes play a crucial role in biological processes including energy and signal transduction, regulation of biological activity, and transport processes. In general, soluble proteins exhibit a hydrophilic surface constituted mainly by polar and charged amino acid side chains. Thus, interactions with membranes are primarily electrostatic in nature and largely restricted to (temporarily) binding of the protein to the periphery of the membrane. However, many of these proteins can also interact with the hydrophobic core of the bilayer which may lead to a partial or a complete integration of the protein into the membrane or which may occur transiently during the transmembrane transport. ${ }^{1}$ Hydrophobic interactions of soluble proteins with membranes are likely to be associated with substantial structural changes in both the protein and the lipid bilayer. However, up to now, the underlying mechanisms are far from being fully understood. Specifically, it is not known which parameters (i) control the interplay between electrostatic and hydrophobic binding and (ii) trigger the structural changes in soluble proteins that are required for the (partial) insertion into the hydrophobic core of bilayer.

Better insight into these processes can be obtained from investigations of model systems that include liposomes and

\footnotetext{
* Address correspondence to this author. Tel.: +49 (0)30 314-21419; fax: +49 (0)30 314-21122; e-mail: hildebrandt@chem.tu-berlin.de.

Max-Planck-Institut für Strahlenchemie.

†niversité Paris.

$\S$ Laboratoire des proteins transtuctrices d'energie.

"Max-Planck-Institut für biophysikalische Chemie.

$\perp$ Technische Universität Berlin.

\# Present address: Leiden University, Huygens Laboratory, Department of Biophysics, Niels Bohrweg 2, 2333 CA Leiden, The Netherlands.
}

structurally well-characterized proteins. The most widely studied model system is constituted by the heme protein cytochrome $c$ $(\mathrm{Cyt}-c)$ and anionic or zwitterionic phospholipids vesicles. ${ }^{2-4}$ During its natural physiological functions, Cyt- $c$ may interact with membranes in quite different ways. As an electron-transfer protein in the respiratory chain of aerobic organisms, Cyt- $c$ transports electrons from cytochrome $c$ reductase to cytochrome $c$ oxidase, which both are transmembrane enzyme complexes. ${ }^{5}$ Most likely, transport between both complexes occurs via lateral diffusion along the mitochondrial membrane such that the positively charged Cyt- $c$ remains continuously in contact with the anionic phospholipids headgroups. In its second function that is related to the activation of an apoptotic pathway, Cyt-c has to be transferred across the mitochondrial membrane and thus through the hydrophobic core of the lipid bilayer. ${ }^{6}$ Hence, both electrostatic and hydrophobic interactions and thus peripheral binding and membrane penetration play a crucial role for the natural functions of this protein.

The present study is dedicated to elucidate the relationship between electrostatic and hydrophobic interactions for binding of Cyt- $c$ to model membranes, the structural consequences for the protein as well as for the phospholipids vesicles. As a model membrane, we have chosen anionic dioleoyl-phosphatidylglycerol (DOPG) vesicles, which can be prepared in a largely monodisperse manner and which have been characterized in detail previously. ${ }^{7}$ On the basis of viscosity and turbidity measurements, we have studied changes of the liposome structure and the liposome-liposome interactions induced by protein binding. Conversely, resonance Raman (RR), UV-vis absorption, and circular dichroism (CD) spectroscopies have been employed to analyze changes in the secondary, tertiary, 
and heme pocket structure as the result of binding to the DOPG liposomes. Combination of these techniques provides a consistent description of the protein-liposome interactions on a molecular and mesoscopic level. The results have an impact not only for a better understanding of the natural functions of Cyt- $c$ but also, in a wider sense, on how soluble proteins in general may interact with biological membranes.

\section{Materials and Methods}

Materials. Horse heart Cyt- $c$ (Sigma, type VI) was dissolved either in $5 \mathrm{mM}$ HEPES/1 mM EDTA (pH 7) or in $2 \mathrm{mM}$ HEPES/1 mM EDTA ( $\mathrm{pH}$ 7.5) buffer for experiments with dioleoylphosphatidylglycerol (DOPG) liposomes. All chemicals used were of highest purity grade available.

Vesicle Preparation. DOPG was purchased from Sigma. Dry films of $10 \mathrm{mg}$ lipid were prepared from a stock solution in chloroform under a nitrogen stream and left under vacuum for at least $8 \mathrm{~h}$ to remove all traces of the organic solvent. The lipid films were re-suspended in HEPES/EDTA buffer and gently vortexed for a few minutes. Large unilamellar vesicles with a diameter of about $150 \mathrm{~nm}$ were then prepared by sequential extrusion through polycarbonate membranes (Osmonics) of decreasing pore size diameter. Vesicle sizes and their distribution as well as vesicle stability were checked by dynamic light scattering (submicron particle analyzer model N4MD, Coulter Electronics).

Binding Assays. Binding to membranes was measured using an ultracentrifugation assay described in detail previously. ${ }^{8}$ Briefly, DOPG dispersions $(1 \mathrm{mg} / 6 \mathrm{~mL})$ were prepared in a 2 $\mathrm{mM}$ Hepes/1 mM EDTA ( $\mathrm{pH}$ 7.5) buffer and brought into contact with Cyt- $c$ at various concentrations. Subsequently, the dispersion was equilibrated overnight. The lipid-protein complexes were separated from the free protein by ultracentrifugation for $4 \mathrm{~h}$ at $4{ }^{\circ} \mathrm{C}(30000-50000 \mathrm{rpm}$; Beckman L7-55). The free protein concentration was determined spectrophotometrically using a molar absorption coefficient of $106.1 \mathrm{mM}^{-1}$ $\mathrm{cm}^{-1}$ at $410 \mathrm{~nm}$. Control experiments with rhodamine 6G labeled DOPG confirmed that no vesicles were present in the supernatant.

UV-Vis Turbidity Experiments. UV-vis spectra were recorded on a Unicam UV2 spectrophotometer with a spectral resolution of $0.5 \mathrm{~cm}^{-1}$, a bandwidth of $4 \mathrm{~nm}$, and a scan speed of $600 \mathrm{~nm} / \mathrm{min}$. Turbidity transients were recorded on a PerkinElmer Lambda2 spectrophotometer at $650 \mathrm{~nm}$. The path length of the optical cell was $10 \mathrm{~mm}$. Cyt- $c$ was added at a rate of 1.4 $\mu \mathrm{L} / \mathrm{min}$ for $3 \mathrm{~h}$ by a perfusor (Braun, Model VI) to $2 \mathrm{~mL}$ solutions of different DOPG concentrations. This rate of Cyt- $c$ addition was considered to be sufficiently slow for equilibration since comparative experiments with slower rates afford very similar results.

Resonance Raman Spectroscopy. RR spectra were measured at ambient temperature with the 413-nm excitation line of a $\mathrm{Kr}^{+}$-laser (Coherent Innova 302) using a spectrograph (U1000, ISA) equipped with a liquid-nitrogen cooled CCD camera. The spectral resolution was $4 \mathrm{~cm}^{-1}$ and the increment per data point was $0.53 \mathrm{~cm}^{-1}$. The power of the incident laser beam focused on the sample was $40 \mathrm{~mW}$. The protein concentration for the RR experiments was $10 \mu \mathrm{M}$.

All RR measurements were repeated several times under the same conditions to ensure reproducibility. To improve the signalto-noise ratio, the individual spectra were added up only if no spectral differences were noted. Prior to the spectra analysis, the structureless background was removed by polynomial

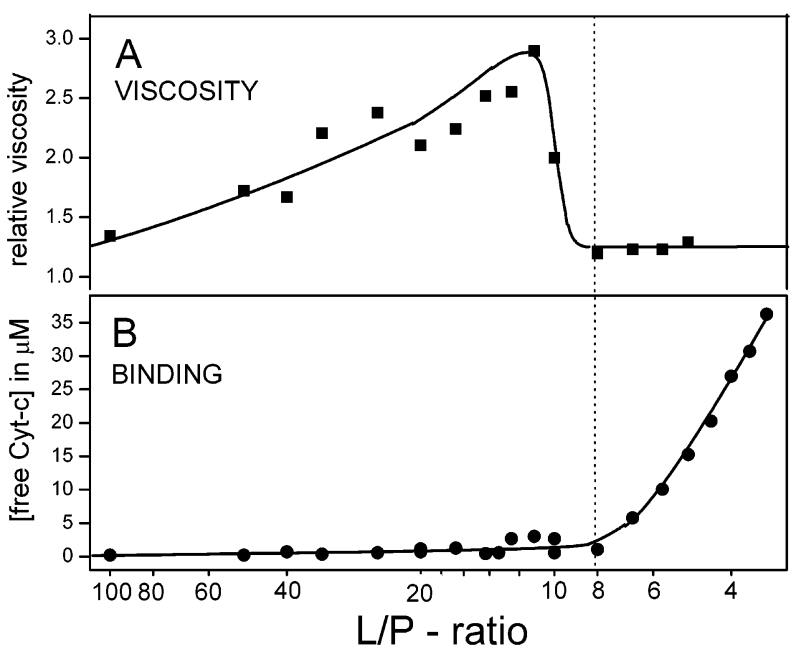

Figure 1. (A) Relative shear viscosity of the lipid/protein dispersion as a function of the DOPG/Cyt $-c$ ratio measured for a constant DOPG concentration of $210 \mu \mathrm{M}$ at an ionic strength of $4.5 \mathrm{mM}$. (B) Binding curve of ferric Cyt-c to DOPG $(210 \mu \mathrm{M})$ at an ionic strength of 4.5 $\mathrm{mM}$.

subtraction. The spectra that were obtained in this way were analyzed by a band fitting or component analysis. ${ }^{9}$

Circular Dichroism Spectroscopy. Spectra were recorded at ambient temperature on a JASCO J-715 spectropolarimeter using a bandwidth of $1 \mathrm{~nm}$, a wavelength increment of $0.5 \mathrm{~nm}$, and a response time of $0.5 \mathrm{~s}$. The optical path length of the quartz cell for far-UV CD $(190-260 \mathrm{~nm})$ and near-UV/Soret CD (250-500 nm) spectroscopic experiments was $1 \mathrm{~mm}$ and $10 \mathrm{~mm}$, respectively. Four scans were averaged per spectrum.

Viscosity Measurements. Viscosities were measured with a Contraves Low Shear 30 viscometer (Stuttgart, Germany) by using a concentric cylinder pendulum (set 1 cup/bob combination). The viscosity is given relative to the viscosity of distilled water obtained under identical conditions.

\section{Results}

Binding Measurements. Figure 1 shows Cyt- $c$ binding to DOPG vesicles as obtained by ultracentrifugation. The DOPG concentration was kept constant at $210 \mu \mathrm{M}$ while the protein concentration was varied. Under the conditions chosen here, the free protein concentration starts increasing when the [DOPG]:[Cyt- $c$ ]-ratio (L/P-ratio) drops below 8:1. Assuming a spherical shape of the protein with a diameter of $3.4 \mathrm{~nm}$, this ratio corresponds to a number of bound Cyt- $c$ that is higher than for a densely packed monolayer covering the vesicles (vide infra). ${ }^{8,10}$

In principle, from such binding measurements the binding constant of Cyt- $c$ to the lipid membranes can be obtained if the ratio $\left[\mathrm{Cyt}-c_{\text {bound }}\right] /\left[\mathrm{Cyt}-c_{\text {unbound }}\right]$ can be determined at low total protein concentration $c_{0}$. However, the data shown in Figure 1 are measured at low ionic strength (ca. $4.3 \mathrm{mM}$ ) and under these conditions nearly all proteins are bound at low $c_{0}$ because of the high electrostatic surface potential of the vesicles. Thus, the concentration of the unbound protein is too low to allow a reliable determination of the binding constant. According to the expression derived by Heimburg and Marsh, ${ }^{8,10}$ the binding constant can be estimated to be $K_{0}=2 \cdot 10^{10} \mathrm{l} / \mathrm{mol}$ under the present conditions.

Viscosity Measurements. Using the same conditions, we have measured relative viscosities of the lipid/protein dispersions (Figure 1). Relative viscosities are obtained by dividing the absolute shear viscosities of the samples by the shear viscosity 

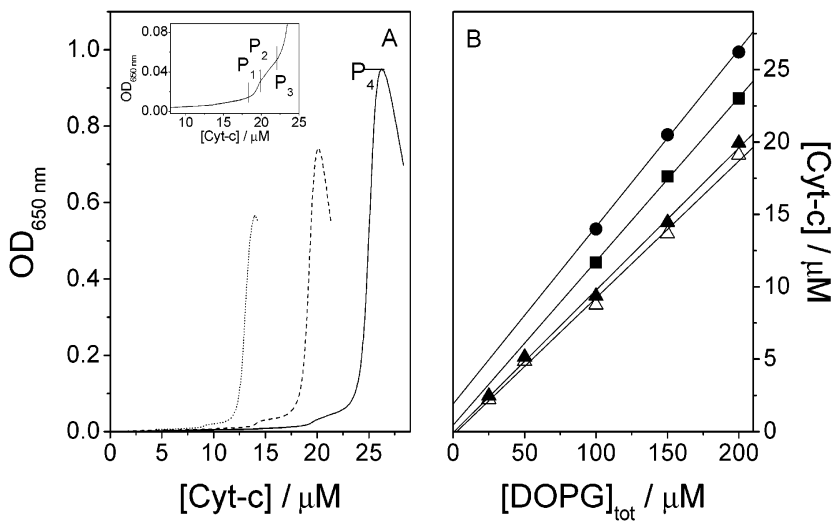

Figure 2. (A) Turbidity transients at $650 \mathrm{~nm}$ measured upon addition of ferric Cyt-c to $100 \mathrm{mM}(\cdots), 150 \mathrm{mM}(---)$, and $200 \mathrm{mM}(-)$ DOPG in $5 \mathrm{mM}$ HEPES/ $1 \mathrm{mM}$ EDTA buffer. Ferric Cyt- $c$ was added at a rate of $1.4 \mu \mathrm{L} / \mathrm{min}$ for $3 \mathrm{~h}$ to $2 \mathrm{~mL}$ solutions of the various DOPG concentrations. The inset in figure A shows a part of the turbidity transient recorded for $200 \mathrm{mM}$ DOPG in an expanded view. The points of characteristic changes in the turbidity $\mathrm{P}_{1}, \mathrm{P}_{2}, \mathrm{P}_{3}$, and $\mathrm{P}_{4}$ are at $\mathrm{L} / \mathrm{P}$ ratios of 10.6, 10.2, 9, and 8, respectively. (B) Characteristic points of turbidity changes obtained from the turbidity transients in $\mathrm{A}$ as a function of the DOPG concentration. The points are marked by the following symbols: $\triangle\left(\mathrm{P}_{1}\right), \boldsymbol{\Delta}\left(\mathrm{P}_{2}\right), \mathbf{\square}\left(\mathrm{P}_{3}\right)$, and $\bullet\left(\mathrm{P}_{4}\right)$.

of water. In the absence of protein, the viscosity of the lipid suspension is close to that of distilled water. With increasing protein concentrations, that is, decreasing L/P-ratios, the viscosity starts increasing up to a value of 3 , indicating long-range interactions between the well-dispersed vesicles. At an L/P ratio of ca. 11:1, the viscosity drastically drops and approaches unity at an L/P-ratio of ca. 8:1.

Turbidity Measurements. Structural changes of liposomes induced by protein binding can be followed by turbidity transients, which indicate changes in the size and structure of the lipid vesicles. ${ }^{11-13}$ Turbidity transients were recorded at 650 $\mathrm{nm}$ such that there is no interference with an electronic transition of Cyt- $c$ and DOPG (vide infra). Independent of the chosen DOPG concentration $(25-200 \mu \mathrm{M})$, all turbidity transients recorded in $5 \mathrm{mM}$ HEPES/ $1 \mathrm{mM}$ EDTA at low ionic strength exhibit the same characteristic shapes, whereas binding of cytochrome $c_{552}$, which exhibits a hydrophobic interaction domain, displays a completely different behavior under the same conditions. ${ }^{14}$ With increasing DOPG concentration the curves are shifted to higher protein concentrations (Figure 2A), indicating that the turbidity is a function of the lipid and the protein concentration. Each turbidity transient recorded at low ionic strength exhibits four characteristic points of turbidity changes. The third and fourth points $\left(\mathrm{P}_{3}\right.$ and $\mathrm{P}_{4}$, respectively) can readily be attributed to flocculation $\left(\mathrm{P}_{3}\right)$ of the DOPG/Cyt- $c$ complex and the subsequent sedimentation of the aggregates $\left(\mathrm{P}_{4}\right)$. Plotting the Cyt- $c$ concentration of the points $\mathrm{P}_{1}-\mathrm{P}_{4}$ versus the corresponding DOPG concentration reveals a linear relationship (Figure 2B) that can be expressed by

$$
[\mathrm{Cyt}-c]_{\mathrm{tot}}=[\mathrm{Cyt}-c]_{\mathrm{unb}}+\frac{1}{R}[\mathrm{DOPG}]_{\mathrm{tot}}
$$

with $R=[\mathrm{DOPG}]_{\mathrm{tot}} /[\mathrm{Cyt}-c]_{\mathrm{b}} \cdot{ }^{13}$ The concentrations of the unbound Cyt- $c$ ([Cyt- $c]_{\text {unb }}$ ) and the DOPG-bound Cyt- $c$ ([Cyt$c]_{\mathrm{b}}$ ) can thus be determined from the intercept and the slope of the curve, respectively. Figure $2 \mathrm{~B}$ shows that $\mathrm{Cyt}-\mathrm{c}$ is bound almost completely to DOPG vesicles at low ionic strength which is in line with the results of binding studies that afford a maximum of $5 \%$ of unbound Cyt- $c$ for the $[\mathrm{DOPG}] /[\mathrm{Cyt}-c]$ ratios (L/P ratios) studied in the turbidity experiments at low ionic strength.

CD and UV-Vis Absorption Spectroscopic Measurements. The far-UV CD spectra of DOPG-bound ferric Cyt- $c$, measured as a function of the L/P-ratio for a constant Cyt- $c$ concentration, do not reveal any significant secondary structure changes. While the ellipticity at $220 \mathrm{~nm}$ remains virtually unchanged, an increase of ellipticity can be observed for the dichroitic minimum at $209 \mathrm{~nm}$, which is essentially independent of the $\mathrm{L} / \mathrm{P}$ ratio (Figure $3 \mathrm{~A}$ ). On the other hand, the near-UV $\mathrm{CD}$ spectra indicate changes of the tertiary structure of the ferric Cyt- $c$. The characteristic minima at 282 and $288 \mathrm{~nm}$, which serve as an indicator for the tight packing of the heme pocket, disappear even for an L/P-ratio as high as 100:1 (Figure 3B). In the Soret $C D$ spectra, the characteristic minimum of the unbound ferric Cyt- $c$ at $417 \mathrm{~nm}$ is converted to a maximum at the same wavelength, while the maximum at $406 \mathrm{~nm}$ is blueshifted to $393 \mathrm{~nm}$. With increasing L/P-ratios, the newly formed minimum at $408 \mathrm{~nm}$ diminishes (Figure 3B).

These tertiary structure changes are accompanied by changes of the heme structure. The UV-vis absorption spectra of the DOPG-bound ferric Cyt- $c$ (data not shown) reveal a slight blueshift of the Soret band absorption maximum to $407.5 \mathrm{~nm}$ for an L/P-ratio of 50. With decreasing L/P-ratio the 695-nm band, which is indicative for the coordination of the native Met- 80 ligand, ${ }^{15}$ disappears and an absorption band at around $620 \mathrm{~nm}$ arises that is characteristic for a ferric high-spin (HS) species.

RR Spectroscopic Measurements. Changes of the Cyt- $c$ heme structure can be studied in more detail by RR spectroscopy. Alterations of the geometry of the porphyrin, the spin and ligation state, and the kind of axial ligands result in characteristic differences in the RR spectra, which allow distinguishing the underlying conformational states of the Cyt$c$. The heme iron of the Cyt-c in aqueous solution $(\mathrm{pH} 7)$ is axially coordinated by His- 18 and Met- 80 and thus is in a sixcoordinated low-spin (6cLS) configuration. This configuration is reflected by the RR marker bands at $1372\left(v_{4}\right), 1502\left(v_{3}\right)$, $1584\left(v_{2}\right)$, and $1635 \mathrm{~cm}^{-1}\left(v_{10}\right)$ (Figure 4D). Binding of ferric Cyt- $c$ to DOPG vesicles, however, results in a shift of all marker band frequencies (Figure $4 \mathrm{~A}-\mathrm{C}$ ) pointing to the formation of a new 6cLS species. The frequency shifts are accompanied by a significant intensity decrease of the $v_{10}$-mode and the complete loss of intensity of the $v_{11}$-mode at $1560 \mathrm{~cm}^{-1}$. Additionally, a broadening of the $v_{3}$ - and $v_{2}$-modes can be observed for high L/P-ratios, which indicates the formation of ferric HS species of Cyt-c (Figure 4).

A global analysis reveals that the RR spectra of ferric Cyt- $c$ bound to DOPG include contributions of three species with altered conformations (denoted as B2) in addition to the native form (state B1). Using a variety of spectroscopic techniques, these species have previously been studied in detail and the axial ligands of the heme iron have been assigned. ${ }^{16}$ Common to all three B2 species is the loss of the native Met- 80 ligand, which is replaced by His-33 (or His-26) and a water molecule in the $6 \mathrm{cLS}$ configuration B2[6cLS] and the six-coordinated high-spin (6cHS) configuration $\mathrm{B} 2[6 \mathrm{cHS}(1)]$, respectively. In the fivecoordinated high-spin (5cHS) form B2[5cHS], the sixth coordination site remains vacant (Figure 5).

Quantitative Analysis of the RR Spectra. Each conformational state of Cyt-c (Table 1) is characterized by its specific RR spectral parameters. These parameters define the respective component spectra that constitute the basis for the quantitative analysis of the RR experiments. Simulating the measured RR spectra by a superposition of these component spectra yields 


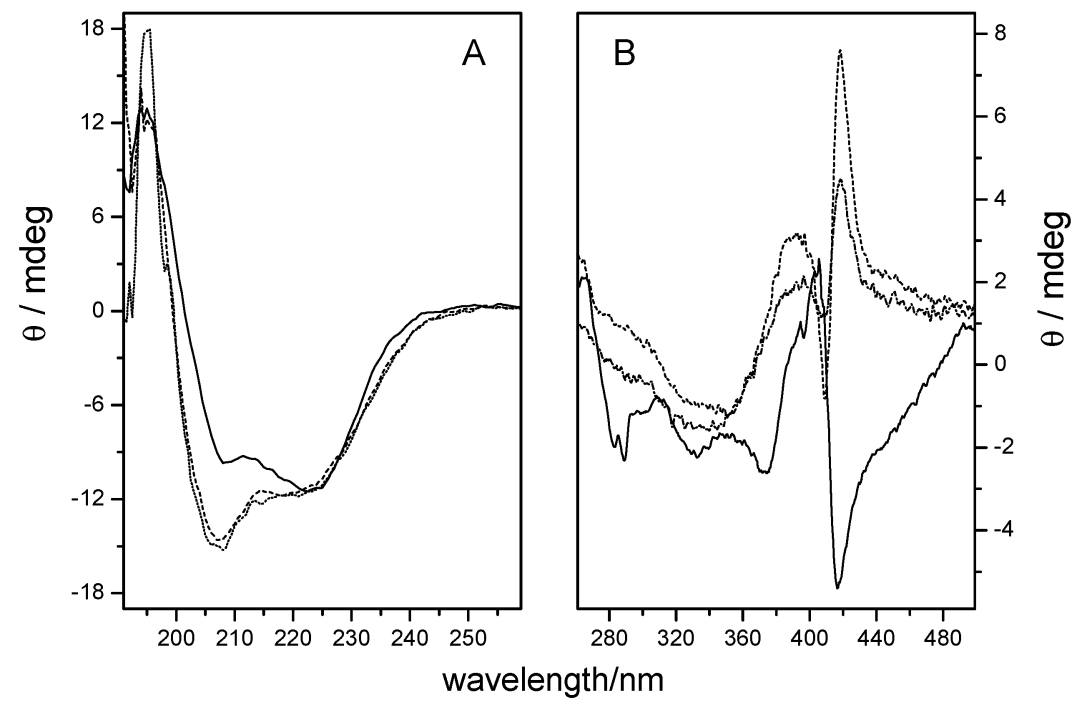

Figure 3. CD spectra of $10 \mu \mathrm{M}$ ferric Cyt- $c$ in $5 \mathrm{mM}$ HEPES/1 mM EDTA buffer (pH 7). (A) Far-UV and (B) near-UV/Soret CD spectra of Cyt- $c$ were obtained in the absence $(-)$ and presence of $250 \mu \mathrm{M}(\cdots)$, and $1 \mathrm{mM}(---)$ DOPG.

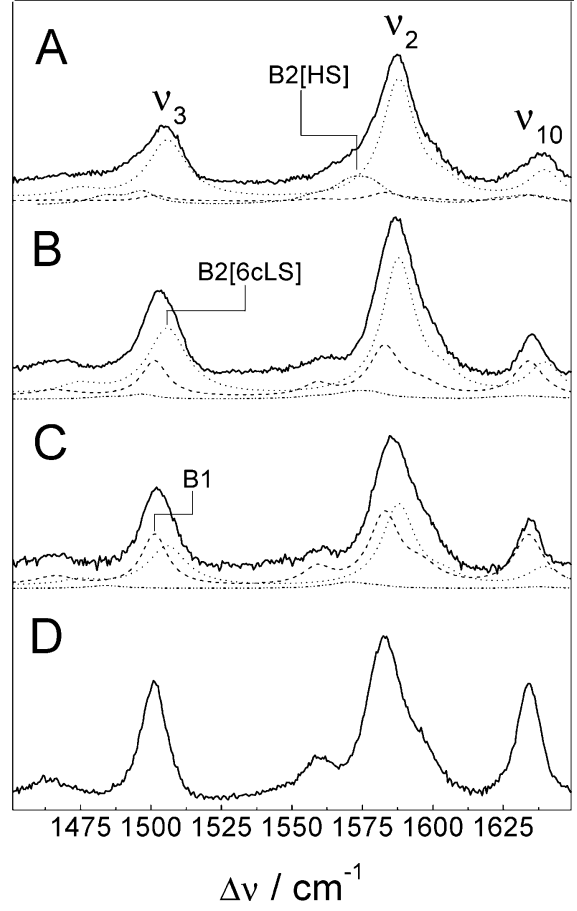

Figure 4. RR spectra of ferric Cyt- $c$ in the marker band region between 1450 and $1650 \mathrm{~cm}^{-1}$. The spectra were recorded with a 413-nm excitation at ambient temperature from $10 \mu \mathrm{M}$ Cyt- $c$ solutions in 5 $\mathrm{mM}$ HEPES/1 mM EDTA at low ionic strength containing (A) 500 $\mu \mathrm{M}$, (B) $140 \mu \mathrm{M}$, (C) $90 \mu \mathrm{M}$, and (D) $0 \mu \mathrm{M}$ DOPG. The component spectra of the various heme configurations are indicated by different line types: B1[6cLS] $(---)$, B2[6cLS] $(\cdots \cdot \cdot)$, and B2[HS] ( $\cdot \cdots \cdot \cdot-)$. $\mathrm{B} 2[\mathrm{HS}]$ represents the sum of the contributions of $\mathrm{B} 2[5 \mathrm{cHS}]$ and $\mathrm{B} 2-$ [6cHS(1)].

the relative spectral contributions of the various species, which can subsequently be converted into relative concentrations using the reciprocal relative RR cross sections determined previously. ${ }^{16}$

Figure 6 shows the relative concentrations of the various species as a function of the L/P-ratio for the experiments at low ionic strength. At the highest applied L/P-ratio, Cyt- $c$ almost exclusively exists in the B2 state with ca. $62 \%, 25 \%$, and $10 \%$ in the $\mathrm{B} 2[6 \mathrm{cLS}], \mathrm{B} 2[6 \mathrm{cHS}(1)]$, and $\mathrm{B} 2[5 \mathrm{cHS}]$ configuration, respectively. Decreasing the DOPG concentration while keeping the Cyt- $c$ concentration constant $(10 \mu \mathrm{M})$ results in a conversion of the B2 species into the native B1 species. Also, the

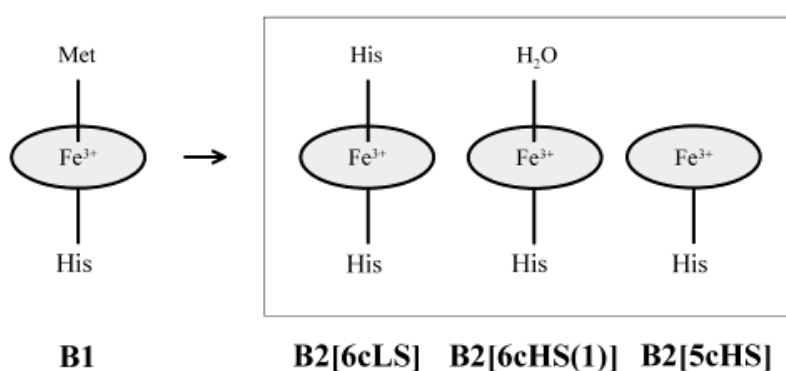

Figure 5. Heme configurations of the various states of ferric Cyt- $c$ bound to DOPG.

distribution among the $\mathrm{B} 2$ species varies with the L/P-ratio. For clarity of the representation in Figure 6, the contributions of $\mathrm{B} 2[5 \mathrm{cHS}]$ and B2[6 $\mathrm{cHS}(1)]$ are added up and presented as B2[HS].

\section{Discussion}

It is obvious from various experimental studies that the binding of Cyt- $c$ to anionic membranes at high degrees of surface coverage results in cooperative events. In titration calorimetry experiments large calorimetric events were observed, which are not present at low degrees of binding. ${ }^{17}$ These authors could show that high degrees of membrane surface coverage exert a lateral pressure on the membrane which can become high enough to overcome the barrier for protein penetration into the membrane. These findings are paralleled by the present results obtained by viscosity and turbidity measurements which display particularly pronounced changes in the concentration range close to saturation coverage of the protein surface (Figures $1,2)$. Conversely, structural changes of the heme pocket occur in the entire range of L/P-ratios but are different below and close to saturation coverage (Figure 6). Thus, it is tempting to relate changes of the vesicle structure and vesicle-vesicle interactions with the mode of Cyt- $c$ binding and the associated structural changes of the protein.

Modes of Protein Binding. Two qualitatively different modes of Cyt- $c$ binding to anionic phospholipid vesicles have been discussed in the literature. Peripheral binding of the protein to the negatively charged phosphatidyl headgroups is attributed to the lysine-rich domain around the exposed heme edge (Figure 7 ) on the front surface of the protein. ${ }^{18,19}$ In such electrostatic 
TABLE 1: Frequencies and Relative Intensities (in Parentheses) of RR Marker Bands of the Various States of Ferric Cyt- $c^{a}$

\begin{tabular}{lllclc}
\hline & & \multicolumn{3}{c}{ modes } \\
\cline { 3 - 5 } species & axial ligands & $v_{4} / \mathrm{cm}^{-1}$ & $v_{3} / \mathrm{cm}^{-1}$ & $v_{2} / \mathrm{cm}^{-1}$ & $v_{10} / \mathrm{cm}^{-1}$ \\
\hline B1[6cLS] & His/Met & $1372.5(1.00)$ & $1501.9(0.26)$ & $1583.6(0.35)$ & $1634.7(0.25)$ \\
B2[6cLS] & His/His & $1374.1(1.00)$ & $1505.8(0.25)$ & $1587.8(0.50)$ & $1639.6(0.12)$ \\
B2[5cHS] & His/- & $1370.3(1.00)$ & $1496.9(0.52)$ & $1576.7(0.55)$ & $1622.5(0.16)$ \\
B2[6cHS(1)] & His/ $\mathrm{H}_{2} \mathrm{O}$ & $1369.5(1.0)$ & $1484.7(0.20)$ & $1569.1(0.44)$ & n.d. ${ }^{b}$
\end{tabular}

${ }^{a}$ Further details were previously given in ref $16 .{ }^{b}$ Not determined.

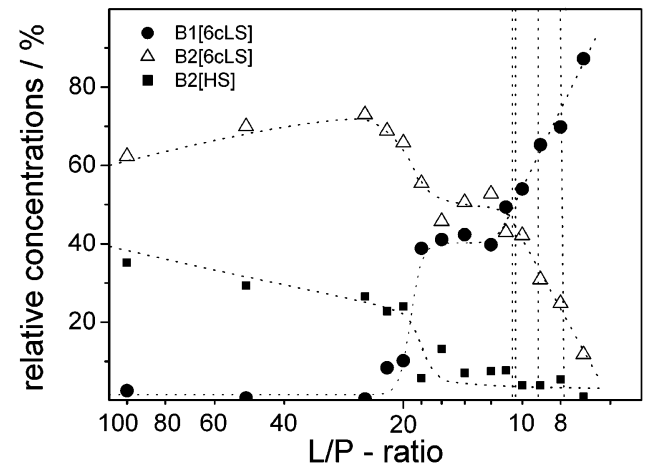

Figure 6. Relative concentrations of the ferric Cyt- $c$ species at ambient temperature in $5 \mathrm{mM}$ HEPES/ $1 \mathrm{mM}$ EDTA buffer as a function of the L/P-ratio. The different Cyt- $c$ species are represented by the following symbols: B1[6cLS] $(\bullet), \mathrm{B} 2[6 \mathrm{cLS}](\triangle)$, and B2[HS] (ם). B2[HS] represents the sum of the contributions of B2[5cHS] and B2[6cHS(1)]. The dotted lines are to guide the eye, while the vertical dashed lines mark the L/P-ratios of the characteristic turbidity changes. The protein concentration was $10 \mu \mathrm{M}$. Further details are given in the text.

complexes, a defined orientation of the protein toward the vesicle surface was suggested. ${ }^{20-22}$ This view was confirmed recently by Kostrzewa et al. who could show that the residues of Lys 72, 86, and 87 point toward the liposomal surface. ${ }^{23}$ Alternatively, a partial penetration of the protein into the bilayer has been found on the basis of EPR studies with lipids selectively labeled with EPR-active probes. In these experiments, signals from spin labels at position C(14) of DOPG were perturbed by Cyt- $c$ binding, pointing to a considerable penetration depth of the protein into the hydrophobic core of the bilayer. ${ }^{8}$ In this concentration range, a substantial loosening of the tertiary structure was inferred from the increased hydrogen/ deuteron exchange rates. ${ }^{24,25}$

Zuckermann and Heimburg ${ }^{17}$ suggested a general mechanism for insertion of soluble proteins into membranes. It was assumed that proteins form a two-dimensional gas on the membrane surface, which generates a high surface pressure at high surface protein concentration. At a critical concentration threshold, this pressure may become high enough for proteins to insert into membranes. This resembles the so-called "carpet" mechanism proposed for pore formation of lytic peptides in membranes. In Cyt- $c$ binding to DOPG, at a critical threshold concentration calorimetric events have been detected that are attributable to protein insertion. ${ }^{17}$ This threshold concentration is identical to that where binding saturation occurs and significant viscosity changes are observed (Figure 1).

Peripheral Binding at Low Surface Coverage. At an L/Pratio of 100 , the bound $\mathrm{Cyt}-c$ is completely converted to the state B2 with a substantial contribution $(\sim 30 \%)$ of the HS forms (B2 [5cHS] + B2[6cHS]). At such high L/P-ratios, electrostatic binding is likely to prevail since no partial penetration of the protein into the bilayer was detectable in EPR experiments. ${ }^{23}$ In fact, the structural changes of the bound Cyt- $c$ are similar to those observed for binding to other types of anionic surfaces

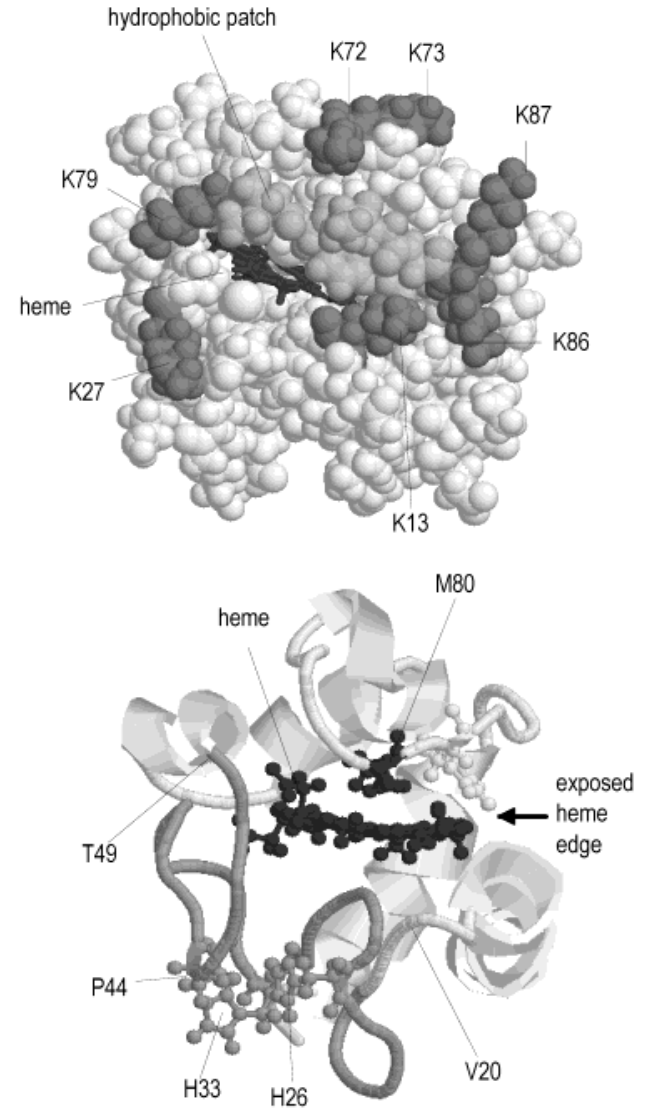

Figure 7. Structure of Cyt- $c$ with view on the front surface (binding domain). The presentation is based on the Brookhaven Databank file "1hrc.pdb". ${ }^{37}$

including micelles and electrodes coated with negatively charged monolayers or anions. ${ }^{26,27}$

According to the UV-CD spectra and in agreement with previous findings, the secondary structure of Cyt- $c$ does not change upon binding to phospholipid vesicles at such high L/Pratios. ${ }^{24,28}$ Hence, it is very likely that the protein retains the spherical shape with a diameter of ca. $34 \AA$. The theoretical saturation coverage of the liposome then corresponds to an L/P ratio of ca. 11 assuming that the phospholipid headgroup of DOPG occupies an area of about $80 \AA^{2}$. At low ionic strength, more than $95 \%$ of the total Cyt-c is bound to DOPG in the linear range of the adsorption isotherm such that the L/P-ratios can be expressed in terms of approximate monolayer coverages. Thus, the L/P-ratio of 100 corresponds to surface coverage of ca. $10 \%$.

Upon lowering the L/P-ratio down to 25 , the HS contribution slightly decreases to about $26 \%$ whereas the $6 \mathrm{cLS}$ form increases (Table 2). Only below the L/P-ratio of 22, the contribution of the native B1 state grows in at the expense of the B2 state. Specifically, the population of the HS species decreases rapidly such that it approaches the limit of detection (ca. 5\%) already at L/P-ratios of $<18: 1$. This tendency reflects the weakening 
TABLE 2: Relationship between Liposome-Protein, Liposome-Liposome Interactions and Structural Changes of Cyt-c as a Function of the L/P-ratio ${ }^{a}$

\begin{tabular}{|c|c|c|c|c|c|c|}
\hline $\mathrm{L} / \mathrm{P}$ ratio & turbidity change & viscosity changes & $\mathrm{B} 1(\%)$ & $\mathrm{B} 2[\mathrm{HS}](\%)$ & $\begin{array}{c}\text { monolayer } \\
\text { formation }(\%)\end{array}$ & mode of binding \\
\hline $100 \rightarrow 20$ & no & steady increase & 0 & $35 \rightarrow 23$ & $10 \rightarrow 50$ & peripheral \\
\hline $20 \rightarrow 18$ & & & $0 \rightarrow 40$ & $23 \rightarrow 5$ & $50 \rightarrow 56$ & \\
\hline $18 \rightarrow 12$ & slight change & steady increase & 40 & 5 & $56 \rightarrow 84$ & peripheral/insertion \\
\hline $12 \rightarrow 10.6$ & P1 & abrupt decrease & $40 \rightarrow 55$ & 4 & $84 \rightarrow 100$ & peripheral second layer/phase separation \\
\hline $10.6 \rightarrow 10.2$ & $\mathrm{P} 2$ & & & & $100 \rightarrow 105$ & \\
\hline $10.2 \rightarrow 9$ & P3 & abrupt decrease & $55 \rightarrow 66$ & 4 & $105 \rightarrow 120$ & second layer/locculation \\
\hline $9 \rightarrow 8$ & P4 & & $66 \rightarrow 72$ & 4 & $120 \rightarrow 135$ & second layer/sedimentation \\
\hline
\end{tabular}

of the electrostatic interactions because of the compensation of the negative headgroup charges on the liposomes when the surface coverage increases from ca. $10 \%$ (L/P-ratio of 100) to ca. $50 \%$ (L/P-ratio of 20). Both the B2/B1 ratio and the HS/LS ratio of state $\mathrm{B} 2$ have been shown to decrease upon lowering the electrostatic field. ${ }^{29}$ In addition, it may be that lateral protein-protein interactions, that have been suggested to stabilize the native state B1, gain importance with increasing coverage of the liposomes.

Partial Insertion of Cyt- $c$ into the Liposomes. At an L/Pratio of $18: 1$, already ca. $40 \%$ of the bound protein is in the state B1. However, this contribution remains constant upon further protein binding up to an L/P-ratio of 12 . This finding suggests that in the range of the surface coverage from ca. $60 \%$ to $85 \%$, a fraction of the bound $\mathrm{Cyt}-c$ is converted to state B2 although electrostatic interactions are steadily weakened with increasing degree of protein binding. Thus, it is concluded that under these conditions, which are high surface coverage and low ionic strength, partial penetration of the protein into the bilayer and formation of hydrophobic contacts in the interior of the bilayer competes with peripheral electrostatic binding as proposed previously. ${ }^{17,19,30,31}$ In fact, it could be shown that hydrophobic interactions of $\mathrm{Cyt}-c$ induce the transition to B2$[6 \mathrm{cLS}] .{ }^{27,32}$ Assuming that the hydrophobically bound Cyt- $c$ is completely converted to B2[6cLS] as it was found for interactions with monomeric SDS, ${ }^{27}$ one can estimate that the maximum of membrane-anchored Cyt- $c$ is ca. $20 \%$ of the total protein at an L/P-ratio of 12. Partial insertion of the protein into the bilayer should result in an expansion of the liposomes which may be related to a slight change in the turbidity at the L/P-ratio of 15 (Figure 2A).

Upon decreasing the L/P-ratio to 11 , the $\mathrm{B} 1$ contribution increases again, implying that binding of further Cyt- $c$ molecules takes place under preservation of the protein structure. Thus, it is concluded that the capacity of the liposomes for (partially) integrated Cyt $c$ is exhausted and peripheral binding dominates again driven by electrostatic interactions which, however, are too weak to cause any structural changes in the protein. The L/P-ratio of 11 corresponds to the full coverage of the surface liposome surface by Cyt- $c$ assuming that the area occupied by an electrostatically bound and hydrophobically achored protein is the same.

Liposome Aggregation and Phase Separation. However, also below this L/P-ratio, DOPG liposomes are capable to bind further Cyt- $c$ molecules (Figure 1). Instead of protein-lipid interaction, now protein-protein interactions have to be taken into account, which result in the formation of a "second" protein layer around the vesicle. Since the increase in size of the Cyt$c / \mathrm{DOPG}$ system by adding a further protein layer can hardly explain the observed significant increase in turbidity $\left(\mathrm{P}_{1}\right.$ at $\mathrm{L} / \mathrm{P}$ $=10.6$ ), this additional protein binding must also affect the shape of the already protein-loaded vesicles. At the L/P-ratio of 10.2 , corresponding to ca. $5 \%$ protein bound in the second monolayer, the turbidity transients flatten again as observed at point $\mathrm{P}_{2}$. This finding indicates that further protein binding does not affect the liposome shape anymore. Instead, the interactions between the protein-loaded vesicles change in this concentration range which may be inferred from the drastic decrease of the viscosity starting at an L/P-ratio of ca. 11 (Figure 1).

This conclusion is confirmed by light microscopic investigations that reveal an increasing connectivity of the vesicles with increasing protein concentration (data not shown). Below the L/P-ratio of ca. 11, vesicular networks that are induced by protein binding span through the entire sample and result in a high viscosity. At lower L/P-ratios, the lipid/protein complexes start to phase separate from the aqueous buffer such that the viscosity decreases. These findings are in line with previous electron microscopy studies on DMPG/Cyt- $c$ complexes which forms clusters at high degrees of protein coverage. ${ }^{33}$

Following this interpretation, phase separation is essentially completed at an L/P-ratio of ca. 9 where viscosity has almost reached the minimum value of 1.0. The subsequent strong increase of the turbidity observed at an L/P-ratio of $9\left(\mathrm{P}_{3}\right)$ is then interpreted in terms of the beginning flocculation of the Cyt- $c$ loaded liposome aggregates. ${ }^{33-35}$ Binding studies in this work as well as previously published results ${ }^{8,36}$ indicate that during the flocculation further Cyt- $c$ can bind to the Cyt-c/ DOPG complexes. The subsequent turbidity decrease at point $\mathrm{P}_{4}(\mathrm{~L} / \mathrm{P}=8)$ is then ascribed to the sedimentation of the flocculated particles.

Hydrophobic Binding Domain. Whereas the lysine residues on the front surface of Cyt- $c$ that form a ring-shape arrangement are known to establish electrostatic binding with anionic surfaces (Figure 7), the identification of those amino acids that serve as an anchor for integrating into the bilayer is less unambiguous. An extended unfolding of the polypeptide chain can be ruled out on the basis of previous FT-IR studies ${ }^{25}$ and of the present far-UV CD spectra which are nearly identical at L/P-ratios of 100 and 12 , corresponding to the minimum (0.0) and maximum $(0.25)$ of the ratio of integrated and peripheral bound protein, respectively. Since the detection limit for changes of the $\alpha$-helical content by CD spectroscopy is estimated to be ca. $3 \%$, the degree of unfolding of the membrane-bound Cyt- $c$ must be less than ca. $12 \%$, corresponding to a decrease of the total percentage of $\alpha$-helix from $40 \%$ of the native, unbound Cyt$\mathrm{c}^{37}$ to at most $37 \%$. Furthermore, an extended unfolding as it may take place upon binding to purely hydrophobic surfaces such as electrodes coated with alkyl-terminated thiols ${ }^{32}$ is associated with the transition to the aquo-coordinated $5 \mathrm{cHS}$ species of the denatured Cyt- $c$ U[5cHS], which is different from that of the His-coordinated B2[5cHS] form. However, the total HS content remains small and unchanged in the L/P-ratio range between 18 and 12. Thus, it is concluded that insertion into the 
bilayer occurs via a relatively small peptide segment without major changes of the overall secondary structure.

There are two hydrophobic patches on the surface of the protein that may serve as a membrane anchor. Both domains are in the vicinity of lysine residues for additional electrostatic interactions with the phospholipid headgroups. Integration of the hydrophobic segment 43-46 into the bilayer and concomitant electrostatic interactions of lysines 22, 39, and 53 would correspond to an orientation of the protein that differs substantially from that of the (purely) electrostatically bound protein. In this case, the front surface of Cyt- $c$ including the heme edge is exposed to the solvent phase as suggested for binding of Cyt- $c$ to zwitterionic vesicles. ${ }^{21}$ However, the formation of B2[6cLS] is difficult to understand for a binding domain remote of the heme pocket.

Such structural changes can plausibly be explained by attributing the membrane-integrating amino acids to the peptide segment from Ile81 to Ile85 on the front surface of the protein which is directly connected to the native Met-80 ligand (Figure 7). Upon displacement and partial insertion of this segment into the hydrophobic core of the bilayer, the coordinative Met-80Fe bond is likely to be weakened and eventually ruptured such that the relatively stable His-33(His-26) adduct of the heme, B2 [6cLS], can be formed. One can readily understand that this mode of binding does not require major changes of the secondary structure which must be ruled out in view of the $C D$ spectroscopic experiments. The hydrophobically bound B2[6cLS] is indistinguishable from the electrostatically bound B2[6cLS] on the basis of RR, SERR, UV-vis absorption, and nearUV CD spectroscopy, indicating the same heme pocket structures in both cases. ${ }^{16}$ Upon Cyt- $c$ insertion via the segment $81-85$, the same lysine residues that are involved in pure electrostatic binding can neutralize the negative charges of the phospholipid headgroups and thus stabilize the membrane-anchored Cyt- $c$ l liposome adduct. Thus, it is likely that the first step of membrane-anchoring is peripheral electrostatic binding which neutralizes the negative charges on the phospholipid headgroups and destabilizes the heme crevice as a prerequisite for the displacement of the hydrophobic peptide segment into the bilayer core. According to this model, the orientation of peripheral bound and integrated Cyt- $c$ with respect to the lipid bilayer surface should be essentially the same.

Even though the hydrophobically or electrostatically induced transition to B2[6cLS] is not associated with major secondary structure changes compared to the native Cyt- $c$, tertiary structure changes must occur inasmuch as a reorientation of the peptide segment $30(20)-49$ is required to bring His-33 (His-26) in proximity to the heme for coordinative binding. As a consequence, the protein surface may be altered also remote from the heme pocket such that it favors the attractive protein-protein interactions and eventually the formation of a second protein layer on the liposomes as it is observed at L/P-ratios below 10 . Aggregation of Cyt- $c$ is not observed in solution at neutral $\mathrm{pH}$ and ambient temperature.

Peripheral Binding versus Partial Penetration. The present study indicates that at low surface coverage electrostatic interactions prevail and peripheral binding of Cyt- $c$ represents the dominant mode of protein-membrane interactions. The strength of electrostatic interactions is reflected by the portions of B2[5cHS] and B2[6cHS] species of the bound Cyt-c, ${ }^{26,27}$ implying that it decreases with increasing L/P-ratio. Evidently, the effective electric field at the protein binding site that promotes the transition to the HS species state does not only depend on the local electrostatic interactions but also on the surface potential of the vesicles that decreases with increasing surface coverage. Once the surface potential is sufficiently low, partial penetration of the protein into the bilayer may effectively compete with peripheral binding. Conversely, a low surface potential, brought about by a high degree of protein binding, can be considered as a crucial parameter for loosening the bilayer structure as a prerequisite for protein integration.

This mechanism may also hold for the transfer of Cyt- $c$ across the mitochondrial membrane which is required for the activation of the Cyt-c-dependent apoptotic pathway. In that case, the initiating event, the weakening of the electrostatic interactions between the protein and the membrane, may be brought about by both a drop in the transmembrane potential and a lowering of the surface membrane potential because of peripheral binding of soluble proteins. Such a mechanism may be of general importance for protein penetration into membranes as it has been shown, for example, in the case of the $\mathrm{C}$-terminal pore-forming domain of the bacteriocin colicin E1. ${ }^{38}$

In the membrane-anchored form, Cyt- $c$ predominantly exists in the B2[6cLS] which is formed upon the exchange of the Met80 by His-33 (His-26). Once the vesicles are completely loaded by peripherally bound and membrane-anchored Cyt- $c$, subsequent protein binding occurs via much weaker forces inasmuch as no protein structural changes are involved, that is, these proteins retain the native structure.

The present findings imply that there are three different modes of Cyt- $c$ binding corresponding to three structurally different protein species.

\section{Conclusions}

At low ionic strength, the mode of Cyt- $c$ binding to DOPG vesicles and the structural changes induced in the protein and liposomes vary with the surface coverage according to the following steps:

(i) At low L/P ratios, Cyt-c binds electrostatically to the DOPG vesicles and is nearly completely converted to the conformationally altered B2 states. Among these states, the relative contributions of the HS species gradually decrease with increasing surface coverage corresponding to a weakening of the electrostatic interactions. There is no observable effect on the vesicles structure, but an increasing connectivity of the liposomes is induced by Cyt-c binding.

(ii) At $\mathrm{L} / \mathrm{P}$ ratios above 18 , partial penetration of Cyt- $c$ into the lipid bilayer takes place, associated with a further strengthening of the long-range liposome-liposome interactions. The hydrophobically bound proteins are converted into the $6 \mathrm{cLS}$ form of B2.

(iii) After monolayer coverage is achieved, additional Cyt- $c$ is bound to vesicle-associated Cyt- $c$ without structural changes in the heme pocket but upon initiating phase separation of the liposomes from the aqueous buffer. This process is followed by further flocculation and subsequent precipitation upon further lowering the $\mathrm{L} / \mathrm{P}$ ratio.

The mutual structural changes in the liposomes and the protein are thus associated with different modes of protein binding that most likely occurs via the same region on the protein surface: electrostatic binding involving the lysine residues surrounding the exposed heme edge and hydrophobic anchoring via the peptide segment $81-85$.

The crucial parameter that controls the interplay between peripheral (electrostatic) and penetrating (hydrophobic) binding appears to be the membrane surface potential which in turn sensitively depends on the protein coverage. 
Acknowledgment. Support by the Deutsche Forschungsgemeinschaft (Sfb 498) to P. H. is gratefully acknowledged.

\section{References and Notes}

(1) Marsh, D.; Horvath, L. I. Biochim. Biophys. Acta 1998, 1376, 267.

(2) de Kruijff, B.; Cullis, P. R. Biochim. Biophys. Acta 1980, 602, 477.

(3) Pinheiro, T. J. T.; Cheng, H.; Seeholzer, S. H.; Roder, H. J. Mol. Biol. 2000, 303, 617.

(4) Tuominen, E. K. J.; Wallace, C. J. A.; Kinnunen, P. K. J. J. Biol. Chem. 2002, 277, 8822

(5) Pettigrew, G. W.; Moore, G. R. Cytochromes c - Biological aspects, 1st ed.; Springer-Verlag: Berlin, Heidelberg, 1987.

(6) Shi, Y. G. Nat. Struct. Biol. 2001, 8, 394.

(7) Ertel, A.; Marangoni, A. G.; Marsh, J.; Hallett, F. R.; Wood, J. M. Biophys. J. 1993, 64, 426.

(8) Heimburg, T.; Marsh, D. Biophys. J. 1995, 68, 536.

(9) Döpner, S.; Hildebrandt, P.; Mauk, A. G.; Lenk, H.; Stempfle, W. Spectrochim. Acta, Part A 1996, 52, 573.

(10) Heimburg, T.; Angerstein, B.; Marsh, D. Biophys. J. 1999, 76, 2575.

(11) Lesieur, S.; Grabielle-Madelmont, C.; Paternostre, M.; Ollivon, M. Anal. Biochem. 1991, 192, 334.

(12) Paternostre, M.; Meyer, O.; Grabielle-Madelmont, C.; Lesieur, S.; Ghanam, M.; Ollivon, M. Biophys. J. 1995, 69, 2476.

(13) Pott, T.; Paternostre, M.; Dufourc, E. J. Eur. Biophys. J. 1998, 27, 237.

(14) Bernad, S.; Oellerich, S.; Soulimane, T.; Noinville, S.; Baron, M. H.; Paternostre, M.; Lecomte, S. Biophys. J. 2004, accepted.

(15) Makinen M. W.; Churg, A. K. Iron Porphyrins; Addison-Wesley Publishing Company: Reading, 1983; Chapter 3, pp 141-219.

(16) Oellerich, S.; Wackerbarth, H.; Hildebrandt, P. J. Phys. Chem. B 2002, 106, 6566 .
(17) Zuckermann, M. J.; Heimburg, T. Biophys. J. 2001, 81, 2458.

(18) Szebeni, J.; Tollin, G. Biochim. Biophys. Acta 1988, 932, 153.

(19) Salamon, Z.; Tollin, G. Biophys. J. 1996, 71, 848.

(20) Spooner, P. J. R.; Watts, A. Biochemistry 1991, 30, 3871.

(21) Pinheiro, T. J. T. Biochimie 1994, 76, 489.

(22) Gorbenko, G. P. Biochim. Biophys. Acta 1999, 1420, 1.

(23) Kostrzewa, A.; Pali, T.; Froncisz, W.; Marsh, D. Biochemistry 2000, 39,6066 .

(24) Muga, A.; Mantsch, H. H.; Surewicz, W. K. Biochemistry 1991, 30,7219 .

(25) Heimburg, T.; Marsh, D. Biophys. J. 1993, 65, 2408.

(26) Murgida, D. H.; Hildebrandt, P. Angew. Chem., Int. Ed. 2001, 40, 728.

(27) Oellerich, S.; Wackerbarth, H.; Hildebrandt, P. Eur. Biophys. J. 2003, 32, 599 .

(28) Pinheiro, T. J. T.; Elöve, G. A.; Watts, A.; Roder, H. Biochemistry 1997, 36, 13122

(29) Murgida, D. H.; Hildebrandt, P. J. Phys. Chem. B 2001, 105, 1578.

(30) Cortese, J. D.; Voglino, A. L.; Hackenbrock, C. R. Biochemistry 1998, 37, 6402.

(31) Gorbenko, G. P.; Domanov, Y. A. Biophys. Chem. 2003, 103, 239.

(32) Rivas, L.; Murgida, D. H.; Hildebrandt, P. J. Phys. Chem. B 2002, $106,4823$.

(33) Heimburg, T.; Biltonen, R. L. Biochemistry 1994, 33, 3, 9477.

(34) Nicholls, P.; Malviya, A. N. Biochem. Soc. Trans. 1973, 1, 372.

(35) Dimitrova, M. N.; Matsumura, H.; Neitchev, V. Z. Langmuir 1997, 13,6516 .

(36) Zhang, F. L.; Rowe, E. S. Biochim. Biophys. Acta 1994, 1193, 219.

(37) Bushnell, G. W.; Louie, G. V.; Brayer, G. D. J. Mol. Biol. 1990, 214,585 .

(38) Zakharov, S. D.; Rokitskaya, T. I.; Shapovalov, V. L.; Antonenko, Y. N.; Cramer, W. A. Proc. Natl. Acad. Sci. U.S.A. 2002, 99, 8654. 